\section{Nach der Tagung ist vor der Tagung}

Wir haben die ereignisreiche Tagung in Aachen unter der phantastischen Präsidentschaft von den Kollegen Jalaie und Rass hinter uns, wir haben dort die persönliche Begegnung nach den Corona-Distanzen, gute Gespräche, interessante Vorträge, die vielen Workshops internationalen Kontakte genossen und schauen nach vorn in einen Herbst / Winter hoffentlich ohne Lockdown und mit weiterer Normalisierung. Den Kollegen Jalaie und Rass sei hier besonders unser Dank ausgesprochen und die Anerkennung, in einem Winter voller Ungewissheiten und Kontaktbeschränkungen so einen vielfältigen Kongress organisiert zu haben! Gratulation!

Nächstes Jahr ist die Jahrestagung für mich persönlich eine ganz besondere, da ich in Hannover die Präsidentschaft übernehme. Für das Motto habe ich mich bei „Choosing wisely“ inspirieren lassen, einer Bewegung die ich seit Jahren beobachte: „Choosing Wisely ist eine 2011 in den USA gestartete Initiative, mit dem Ziel, unnötige medizinische Leistungen zu reduzieren. “ Bertelsmannstiftung, https://www.bertelsmannstiftung.de/de/unsere-projekte/patientmit-wirkung/projektthemen/choosing-wisely/.

In der Inneren Medizin ist mir das Thema Choosing Wisely bereits in der Palliativmedizin und der Nephrologie im Rahmen von Weiterbildungen vor mehreren Jahren begegnet und positiv aufgefallen. Je mehr Therapie-Vielfalt, desto wichtiger wird es, für jedes Krankheitsbild unabhängige Hilfestellungen für „Kluge Entscheidungen“ zur Verfügung zu haben. Die Phlebologie zeichnet sich dadurch aus, dass die Therapie fast nie ein Notfall ist, sodass man nach Aufklä- rung den Patienten in dieses Entscheidungs-Boot mit hineinnehmen kann. Sollten wir nicht über alle Therapieoptionen an unserem Haus / unserer Praxis verfügen, können wir weitere Kollegen hinzuziehen. Daher ist der Titel „Gemeinsam klug entscheiden“ das Motto der Kommenden Jahrestagung in Hannover, zu der ich schon jetzt recht herzlich einladen möchte. Ich würde mich über Vorschläge und Beiträge zu diesem Thema aus der Mitte unserer Gesellschaft sehr freuen.

Und natürlich hoffe ich schon jetzt, dass sich jeder das Datum im Kalender freihält! Mittwoch, der 28.9. - Samstag der 1.10.2022 freue ich mich auf Sie in Hannover!

Ihre Erika Mendoza 